\title{
Reversible optical nonreciprocity in periodic structures with liquid crystals
}

\author{
Andrey E. Miroshnichenko, ${ }^{1, a)}$ Etienne Brasselet, ${ }^{2, b)}$ and Yuri S. Kivshar ${ }^{1}$ \\ ${ }^{1}$ Nonlinear Physics Centre and Centre for Ultra-high bandwidth Devices for Optical Systems (CUDOS), \\ Australian National University, Canberra ACT 0200, Australia \\ ${ }^{2}$ Centre de Physique Optique Moléculaire et Hertzienne, Université Bordeaux 1, CNRS, 351 Cours de la \\ Libération, 33405 Talence Cedex, France
}

(Received 24 November 2009; accepted 7 January 2010; published online 10 February 2010)

\begin{abstract}
We demonstrate how to achieve reversible nonreciprocal optical response in a periodic photonic structure with a pair of defects, one of them being a nonlinear liquid crystal defect layer. The twin defect structure is symmetric at low intensity and becomes asymmetric above an intensity threshold that corresponds to the optical reordering of the liquid crystal. We show that nonreciprocal effects can be reversed by changing the wavelength as a consequence of the wavelength dependent light localization at the defect mode inside the structure. (c) 2010 American Institute of Physics.
\end{abstract}

[doi:10.1063/1.3300824]

Optical nonreciprocity (ONR) refers to different properties for opposite propagation directions of electromagnetic waves. Nonreciprocal response is usually related to timereversal symmetry breaking of light-matter interaction. ${ }^{1} \mathrm{~A}$ well-known example is found in gyrotropic materials, where left- and right-handed polarized fields propagate at different speeds in the presence of an external static magnetic field. ${ }^{2-4}$ However, ONR is not restricted to the use of magnetic field. Indeed, it has been suggested that time-dependent refractive index modulation can lead to ONR by inducing dynamically indirect interband photonic transitions. ${ }^{5}$ Nonreciprocity can also be achieved without the use of applied external fields in spatially asymmetric light sensitive media, ${ }^{1}$ and, formally, one can make the distinction between linear and nonlinear systems. The former class is based on optical absorption ${ }^{6}$ or anisotropy, ${ }^{7}$ or employs optomechanical effects. ${ }^{8}$ On the other hand, the use of optical nonlinearities allows to achieve tunable devices driven by the light itself. For example, power dependent ONR has been proposed for the realization of alloptical diodes $^{9-11}$ and unidirectional couplers. ${ }^{12}$

Among various optically nonlinear materials, liquid crystals (LCs) possess orientational third-order nonlinearities that are up to several orders of magnitude larger than Kerr electronic nonlinearities of conventional dielectrics. ${ }^{13}$ Therefore, the efficiency of all-optical nonreciprocal strategies based on LCs can be enhanced in dielectric periodic structures by embedding a LC defect layer asymmetrically inside it. Such a geometry has been previously explored ${ }^{14}$ but for an hypothetical LC material exhibiting a first-order optical Frédericksz transition (OFT) in the absence of periodic structure, ${ }^{15}$ and in the particular case $\lambda=\lambda_{\mathrm{d}}$ where $\lambda$ is the wavelength of light and $\lambda_{d}$ is a defect mode wavelength. In fact, as shown here, such a nonreciprocal behavior is more general and applies to common nematic LCs, whatever $\lambda$ is. Nevertheless, the corresponding optical diode behavior is intrinsically unidirectional. In this Letter, we propose a strategy enabling all-optical diode operation to work both for light propagation toward $\pm z$, thus introducing the concept of all-optical reversible nonreciprocity, which is controlled by the wavelength in the present case.

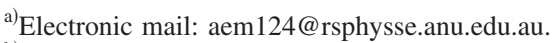

b) e.brasselet@cpmoh.u-bordeaux1.fr
}

For the purpose of demonstration, we consider a photonic structure made of alternating layers of $\mathrm{SiO}_{2}$ and $\mathrm{TiO}_{2}$ with thicknesses 103 and $64 \mathrm{~nm}$, respectively, whose transmission spectrum has a gap in the visible range between 500 and $720 \mathrm{~nm}$. Inside such a structure either one (case I) or two (case II) defect layers are embedded. In case I, the defect is a nematic layer with its director (i.e., the unit vector $\mathbf{n}$ that represents the local average molecular orientation of the LC) lying along the light propagation axis $z$ in the absence of reorientation, $\mathbf{n}_{0}=\mathbf{e}_{z}$ [see Fig. 1(a)]. Moreover, the number of $\mathrm{SiO}_{2} / \mathrm{TiO}_{2}$ building blocks differs for the left and right side, $N_{\mathrm{L}} \neq N_{\mathrm{R}}$ [see Fig. 1(b)]. In case II there is an additional, optically linear, defect layer with the same optical path length than the liquid crystal one, but with $N_{\mathrm{L}}=N_{\mathrm{R}}$ [see Figs. 1(d) and 1(e)].

The optical response of these two systems under a linearly polarized light is obtained following the standard Berreman's $4 \times 4$ matrix formalism and taking into account the orientational optical nonlinearities of the LC layer. ${ }^{16-18}$ The calculations are performed for a generic LC with the refractive indices $n_{\perp}=1.5$ and $n_{\|}=1.7$, where " $\perp$ " and "||" refer to a direction perpendicular and parallel to $\mathbf{n}$, respectively. Moreover, the ratio between splay and bend Frank elastic constants is taken as $K_{1} / K_{3}=2 / 3 .{ }^{19}$ We choose defect layer thicknesses $L=5 \mu \mathrm{m}$ for both cases, which means that the linear defect layer in case II has a refractive index $n_{\perp}$.

In case I, the optical field distribution inside the unperturbed photonic structure is asymmetric, as depicted in Fig. 1(b) where a typical calculated electric field profiles are shown for left-to-right (LR) and right-to-left (RL) incident light. Obviously, the light confinement inside the LC layer depends on the propagation direction. Indeed, in the example shown in Fig. 1(b), the localization of light is stronger in the LR case than in the RL one. Therefore, the light intensity threshold above which optical reordering occurs differs for the LR and RL situations. The corresponding normalized thresholds $\rho_{\text {th }}$ are shown in Fig. 1(c) as a function of the wavelength of light $\lambda$, where we introduce the normalized incident intensity with respect to the OFT value for a LC slab alone, $\rho=I_{\text {incident }} / I_{\mathrm{OFT}}$. As expected, these thresholds are spectrally modulated with local minima at each of the defect mode frequency ${ }^{17}$ and we find that $\rho_{\text {th }}^{\mathrm{LR}}<\rho_{\text {th }}^{\mathrm{RL}}$ independently of $\lambda$ [see Fig. 1(c)]. The optical response of the whole 
(a)

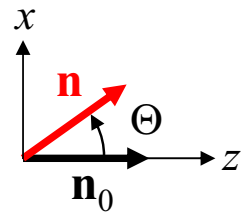

(b)

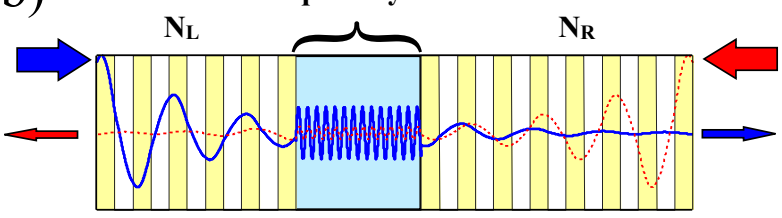

(c)

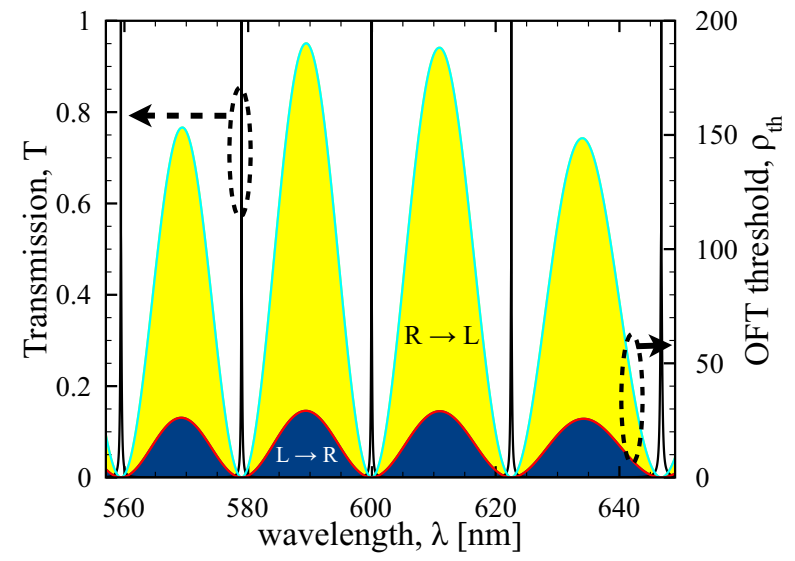

Liquid crystal Linear layer

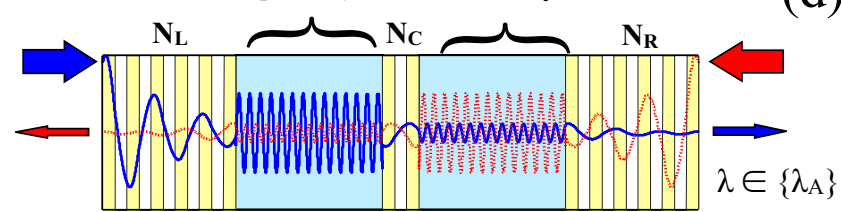

(d)

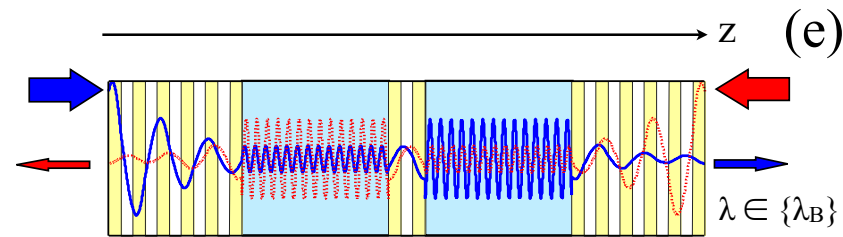

(e)

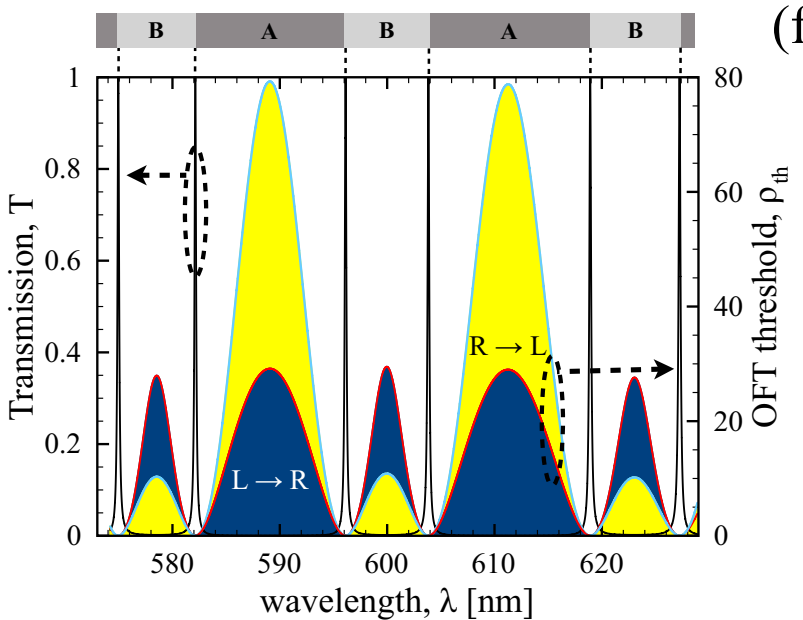

(f)

FIG. 1. (Color online) (a) Director reorientation angle $\Theta(z)$ with respect to the unperturbed state $\mathbf{n}_{0}=\mathbf{e}_{z}$, which satisfies the boundary conditions $\Theta(z=0, L)=0$. (b) Case I: asymmetric single LC defect periodic structure with a different number of $\mathrm{SiO}_{2} / \mathrm{TiO}_{2}$ periodic cells on the left and right sides, $N_{\mathrm{L}} \neq N_{\mathrm{R}}$. Typical electric field amplitude profile for left-to-right light propagation, "LR," (respectively, right- to-left, "RL") in the absence of reorientation. (c) Transmission $T$ and normalized threshold intensity $\rho_{\text {th }}^{\mathrm{LRL}}$ with $N_{\mathrm{L}}=5$ and $N_{\mathrm{R}}=7$ for case I. Right part, case II: [(d) and (e)] Electric field profile within the unperturbed twin defect layer structure for LR and RL propagation and a wavelength belonging to region referred to as "A" [panel (d)] or "B" [panel (e)]. (f) Transmission $T$ and normalized threshold intensity $\rho_{\text {th }}^{\mathrm{LR}, \mathrm{RL}}$ with $N_{\mathrm{L}}=N_{\mathrm{R}}=5$ and $N_{\mathrm{C}}=1$.

structure is thus reciprocal for $\rho<\rho_{\mathrm{th}}^{\mathrm{LR}}$ and nonreciprocal for $\rho>\rho_{\text {th }}^{\mathrm{LR}}$ following the generic scheme relying on nonlinearity. ${ }^{9-11}$ The unidirectionality of such optical diodes, however, is imposed by the intrinsic asymmetry of the structure that is present even when the nonlinearity is not activated.

From above considerations, any attempt to control the unidirectionality thus requires an initially symmetric device. The nonreciprocal condition, however, imposes a nonlinear asymmetry. Such conditions are satisfied in the case II, where a nonlinear/linear pair of defects is embedded into a symmetric periodic structure, $N_{\mathrm{R}}=N_{\mathrm{L}}$, and separated by a symmetric multilayer characterized by $N_{\mathrm{C}}$ [see Figs. 1(d) and 1(e)]. The LR and RL normalized reorientation thresholds are shown in Fig. 1(f). We find that either $\rho_{\mathrm{th}}^{\mathrm{LR}}<\rho_{\mathrm{th}}^{\mathrm{RL}}$ or $\rho_{\mathrm{th}}^{\mathrm{LR}}$ $>\rho_{\mathrm{th}}^{\mathrm{RL}}$ condition is satisfied depending on the wavelength, as illustrated by the spectral zones referred to as "A" and "B," respectively [see Fig. 1(f)]. As a matter of fact, such a behavior is consistently retrieved from the spatial confinement of the incident light field into the unperturbed LC defect layer. This is illustrated in Figs. 1(d) and 1(e) where the typical electric field calculated profiles are shown for the LR and RL situations in the case A, where confinement is more efficient for LR propagation [see Fig. 1(d)], and in the case $\mathrm{B}$, where RL confinement is better [see Fig. 1(e)].

As a result, ONR unidirectionality can be reversed by changing the wavelength of light. Note, however, that the spatial mirror symmetry for the light field is restored when the wavelength of the incident light matches one of the defect mode wavelength $\lambda_{\mathrm{d}}$, which results in identical thresholds $\rho_{\mathrm{th}}^{\mathrm{LR}}\left(\lambda_{\mathrm{d}}\right)=\rho_{\mathrm{th}}^{\mathrm{RL}}\left(\lambda_{\mathrm{d}}\right)$.

When $\min \left[\rho_{\mathrm{th}}^{\mathrm{LR}, \mathrm{RL}}(\lambda)\right]<\rho<\max \left[\rho_{\mathrm{th}}^{\mathrm{LR}, \mathrm{RL}}(\lambda)\right]$ at a given wavelength, the LC is reoriented for light impinging from, i.e., $\mathbf{n} \neq \mathbf{n}_{0}$, from one direction, whereas $\mathbf{n}=\mathbf{n}_{0}$ for the opposite light propagation direction. Above $\max \left[\rho_{\mathrm{th}}^{\mathrm{LR}, \mathrm{RL}}(\lambda)\right]$, the LC is always reoriented for any direction of the incident light propagation, however, the LR and RL reoriented states remain distinct, thus preserving a nonreciprocal material response.

Also, we note that the intensity is not the only parameter that dictates the optically induced reorientation qualitative behavior. Indeed, it is known that the detuning parameter $\delta$ $=\lambda-\lambda_{\mathrm{d}}$ between the incident wavelength and the nearest defect mode wavelength controls the order of the OFT, which is first-order for $\delta>0$ and second-order when $\delta<0 .{ }^{17,18}$ The ONR behavior associated with LC reorientation is summarized in Fig. 2 where we plot (i) the phase associated to LC reorientation for the transmmitted light, $\varphi_{\mathrm{t}}$ (i.e., $\varphi_{\mathrm{t}}=0$ if $\mathbf{n}$ $=\mathbf{n}_{0}$ and (ii) the reduced amplitude of the output Poynting vector, $\widetilde{S}_{z}^{\text {out }}=\operatorname{sign}\left(k_{z}\right) T$, that are represented as a function of $\widetilde{S}_{z}^{\text {in }}=\operatorname{sign}\left(k_{z}\right) \rho$, where $\mathbf{k}= \pm(2 \pi / \lambda) \mathbf{e}_{z}$ is the wave vector of the incident light. In this figure, both positive [see Figs. 2(a) and 2(b)] and negative [see Figs. 2(c) and 2(d)] detuning 


$$
\begin{gathered}
\delta=+1 \mathrm{~nm} \\
-\lambda_{\mathrm{A}}=605 \mathrm{~nm} \\
--\lambda_{\mathrm{B}}=597 \mathrm{~nm}
\end{gathered}
$$

(a)

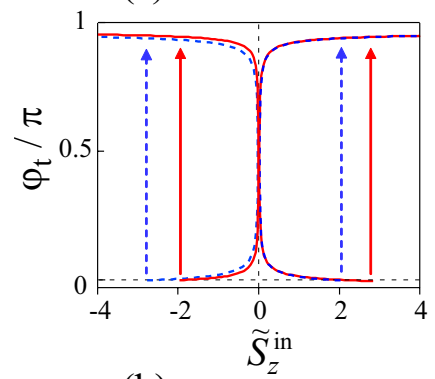

(b)

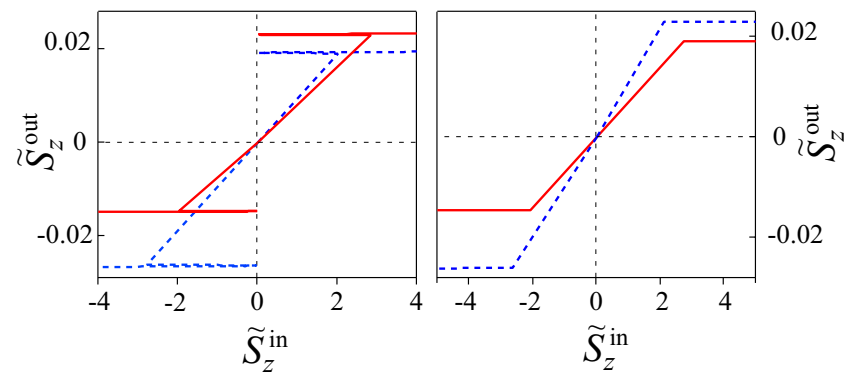

FIG. 2. (Color online) Phase jump of the transmitted light, $\varphi_{t}$, and output Poynting vector amplitude normalized to its threshold value, $\tilde{S}_{z}^{\text {out }}$ $=\operatorname{sign}\left(\mathrm{k}_{\mathrm{z}}\right) T$, vs. $\widetilde{S}_{z}^{\text {in }}$ for positive (a) and (b) and negative (c) and (d) values of $\delta=\lambda-\lambda_{\mathrm{d}}$ in the vicinity of the two adjacent defect modes $\lambda_{\mathrm{d}} \simeq 604 \mathrm{~nm}$ and $\lambda_{\mathrm{d}} \simeq 596 \mathrm{~nm}$. Solid and dashed curves refer to wavelength belonging to region A and B respectively, see Fig. 1(f).

values in the vicinity of the two adjacent defect modes $\lambda_{\mathrm{d}}$ $\simeq 604 \mathrm{~nm}$ and $\lambda_{\mathrm{d}} \simeq 596 \mathrm{~nm}$ [see Fig. 1(f)] are shown. Note that $\widetilde{S}_{z}^{\text {in }}>0$ for the light propagating in LR direction, and $\widetilde{S}_{z}^{\text {in }}<0$ for opposite direction (RL). In Fig. 2, the solid and dashed curves refer to wavelength belonging to region $\mathrm{A}$ and B, respectively [see Fig. 1(f)].

We find a reversible ONR response both for the positive and the negative detuning situations. However, the case $\delta>0$ looks more attractive for both phase and amplitude dependencies with respect to the propagation direction and intensity of the incident field. First, the phase of the transmitted field, $\varphi_{\mathrm{t}}$, typically experiences a $\pi$-jump at the LC reorientation threshold [see arrows in see Fig. 2(a)]. This is due to the fact that the defect mode resonance shifts toward larger wavelength when the OFT occurs. Therefore, the fixed excitation wavelength $\lambda$ passes from one to the other side of the defect mode resonance due to reorientation, i.e., the effective detuning changes it sign, which is accompanied by a $\pi$ phase shift. Second, the ratio of the transmission in opposite directions, $T^{\mathrm{LR}} / T^{\mathrm{RL}}$ or $T^{\mathrm{RL}} / T^{\mathrm{LR}}$ depending on the wavelength, of the order of 100 is achieved in the hysteresis regions for positive detuning $\delta=+1 \mathrm{~nm}$ [see Fig. 2(b)], as shown in Fig. 3. Practically, this ratio can be further enhanced by using larger number of periodic layers $N_{\mathrm{R}, \mathrm{L}}$.

In conclusion, we have proposed a simple approach to achieve a reversible nonreciprocal optical response con-
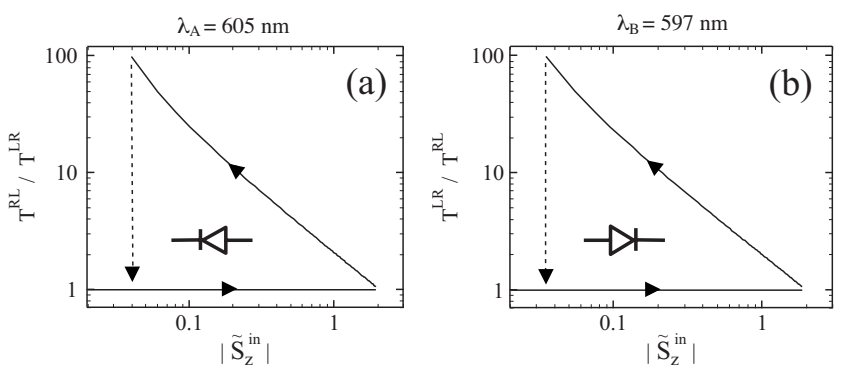

FIG. 3. Ratio of the transmission coefficient in opposite directions (a) $T^{\mathrm{RL}} / T^{\mathrm{LR}}$ for wavelength $\lambda_{A}=605 \mathrm{~nm}$, and (b) $T^{\mathrm{LR}} / T^{\mathrm{RL}}$ for wavelength $\lambda_{B}$ $=597 \mathrm{~nm}$, which both are associated to a hysteresis behavior for positive detuning $\delta=+1 \mathrm{~nm}$ as shown in Fig. 2(b). Both plots are nearly identical, which illustrates the reversibility of the ONR unidirectionality.

trolled by the wavelength of light. This has been made possible by using a linear/nonlinear pair of defect layers embedded in a periodic structure that is invariant by mirror symmetry when the nonlinearity is not activated. For the purpose of illustration, liquid crystals have been used as the optically nonlinear material, however our approach basically works whatever the nature of the nonlinearity. Moreover, the present concept is not restricted to one-dimensional structures and can be easily generalized to two-dimensional and three-dimensional geometries.

The work has been supported by the Australian Research Council project DP0771218 and the the France-Australia cooperation project 21337 of CNRS.

${ }^{1}$ R. J. Potton, Rep. Prog. Phys. 67, 717 (2004).

${ }^{2}$ A. Zvezdin and V. Kotov, Modern Magnetooptics and Magnetooptical Materials (Taylor \& Francis, London, 1997).

${ }^{3}$ A. Figotin and I. Vitebsky, Phys. Rev. E 63, 066609 (2001).

${ }^{4}$ A. B. Khanikaev, A. V. Baryshev, M. Inoue, and Yu. S. Kivshar, Appl. Phys. Lett. 95, 011101 (2009).

${ }^{5}$ Z. Yu and S. Fan, Nat. Photonics 3, 91 (2009).

${ }^{6}$ G. S. Agarwal and S. D. Gupta, Opt. Lett. 27, 1205 (2002)

${ }^{7}$ J. Hwang, M. H. Song, B. Park, S. Nishimura, T. Toyooka, J. W. Wu, Y. Takanishi, K. Ishikawa, and H. Takezoe, Nature Mater. 4, 383 (2005).

${ }^{8}$ S. Manipatruni, J. T. Robinson, and M. Lipson, Phys. Rev. Lett. 102, 213903 (2009).

${ }^{9}$ S. F. Mingaleev and Y. S. Kivshar, J. Opt. Soc. Am. B 19, 2241 (2002).

${ }^{10}$ H. Zhou, K.-F. Zhou, W. Hu, Q. Guo, S. Lan, X.-S. Lin, and A. V. Gopal, J. Appl. Phys. 99, 123111 (2006).

${ }^{11}$ X.-S. Lin, J.-H. Yan, L.-J. Wu, and S. Lan, Opt. Express 16, 20949 (2008).

${ }^{12}$ A. Alberucci and G. Assanto, Opt. Lett. 33, 1641 (2008)

${ }^{13}$ I. C. Khoo, Phys. Rep. 471, 221 (2009).

${ }^{14}$ A. E. Miroshnichenko, I. Pinkevych, and Yu. S. Kivshar, Opt. Express 14, 2839 (2006)

${ }^{15}$ N. V. Tabiryan, A. V. Sukhov, and B. Y. Zel'dovich, Mol. Cryst. Liq. Cryst. 136, 1 (1986).

${ }^{16}$ D. W. Berreman, J. Opt. Soc. Am. 62, 502 (1972).

${ }^{17}$ A. E. Miroshnichenko, E. Brasselet, and Yu. S. Kivshar, Appl. Phys. Lett. 92, 253306 (2008)

${ }^{18}$ A. E. Miroshnichenko, E. Brasselet, and Yu. S. Kivshar, Phys. Rev. A 78 , 053823 (2008).

${ }^{19}$ Only elastic reorientation modes lying in the plane defined by the electric field and the wavevector of the incident light are considered within a plane wave description of the optical field. Therefore, the twist elastic constant $K_{2}$ is not involved in the equations of the model. 